\title{
Prospects of Advanced Polymer Research in Industry
}

\author{
Geoffrey ALLEN
}

UNILEVER PLC-U.K.

(Received August 20, 1986)

\begin{abstract}
In the last decade the high tonnage polymers which form the base of the polymer industry have reached a plateau. Difficult market conditions have led to rationalization and caused chemical companies to refine their commodity products to gain a competitive edge. New grades of well-established polymers with tighter profiles of properties continue to emerge. Thus, there will be a steady evolution of more advanced products even in this mature market. Much has been done to control the microstructure of polymers and increased attention will be given to control 'of macrostructures. Composite materials now enjoy a good range of high modulus fibres and a range of new matrices, but fibre management in the lay-up of the composite is still a problem. At the other extreme is the production of polymers with controlled porosity for separation systems, catalyst supports or ultra-lightweight products. Polymerization of monomers dispersed in emulsions makes such structures feasible. Low volume speciality polymers are now making a significant contribution to the profitability of the industry. Some $25 \%$ of the industry's profit comes from speciàlity polymers which account for less than $10 \%$ by weight of the products sold. Naturally, these advanced materials attract increasing attention. Speciality polymers with good engineering properties, special surface effects or opto-electronic properties are still seen as the most exciting materials.
\end{abstract}

KEY WORDS Polymer Research / Structured Polymers / Modified Natural

Polymers / Conducting Polymers / Thin Film Polymers / Hybrid Polymers /

A wide ranging interpretation of 'advanced polymer research' in this plenary lecture enables a survey to be made of some current developments of polymer systems which will find their place in industrial usage in the next decade. Likely progress is examined in:

(i) high tonnage polymers,

(ii) polymer systems incorporating macroscopic structures, e.g., fibre re-inforced composites, foams,

(iii) modification of natural polymers, and of course,

(iv) speciality polymers.

No attempt is made to be complete, instead a broad ranging 'pot-pourri' of possibilities emerging from current $\mathrm{R} \& \mathrm{D}$ is presented.

\section{HIGH TONNAGE POLYMERS}

In the last decade the growth in markets for the high-tonnage polymers such as polystyrene, ABS, polyethylene, polypropylene, PVC and polyethylene teraphthlate and nylon has diminished after the boom years of petrochemicals. Difficult market conditions have led to rationalization in the industry and caused companies to refine the specification, even of some of their low-priced commodity products in order to gain a competitive edge. New grades of well-established products, with tighter control of the profile of properties, are emerging. These new products in themselves result from advanced polymers research they require control of the linearity and microstructure of the polymer chain and of the molecular weight distribution. 
G. Allen

Table I. Properties of representation grades of polyethylene

\begin{tabular}{lccccc}
\hline & VLDPE (1) & VLDPE (2) & LDPE & LLDPE & HDPE \\
\hline Density $\left(\mathrm{g} \mathrm{cm}^{-3}\right)$ & 0.900 & 0.906 & 0.920 & 0.920 & $0.94-0.97$ \\
$\bar{M}_{n} \times 10^{-5}$ & 0.31 & 0.19 & 0.24 & 0.28 & - \\
$\bar{M}_{w} \times 10^{-5}$ & 1.37 & 1.55 & 2.12 & 1.14 & - \\
Secant modulus $\left(1 \mathrm{lb} / \mathrm{in}^{2}\right)$ & 13 & 17 & 21 & 30 & 120 \\
Melting point $/{ }^{\circ} \mathrm{C}^{\mathrm{a}}$ & 116 & 118 & 108 & \\
\hline
\end{tabular}

a Measured by differential scanning calorimetry.

The linear low density (LLDPE) and very low density (VLDPE) variants of polyethylene, as developed by Union Carbide Corporation, are illustrative. ${ }^{1}$ They add to low density polyethylene (LDPE) produced by high pressure technology and the medium and high density (HDPE) products obtained from ZeiglerNatta type process technology, which has been available for many years. LDPE (density 0.915 to $0.930 \mathrm{~g} \mathrm{~cm}^{-3}$ ) has a highly branched chain structure, and HDPE (density 0.94 to 0.97 $\mathrm{g} \mathrm{cm}^{-3}$ ) incorporates a few long chain branches in each molecule. The new LLDPE and VLDPE grades are made by copolymerizing ethylene with $\alpha$-olefin monomers, the random incorporation of this short-chain branching in essentially linear chains yields to densities in the range $0.900-0.930 \mathrm{~g} \mathrm{~cm}^{-3}$.

The properties of these new grades are nicely segmented as shown in Table I.

The degree of crystallinity increases in a moulded specimen as the density increases, but the melting point as measured by differential scanning calorimetry does not change markedly, i.e., $116-120^{\circ} \mathrm{C}$. The melting points of LDPE's of similar densities are some $10-12^{\circ} \mathrm{C}$ lower. The modulus of LLDPE and VLDPE lie on either side of conventional LDPE. Figure 1 shows that the LLDPE and VLDPE variants of polyethylene have altogether cleaner molecular weight distribution than LDPE.

Thus, even in commodity polymers a range of discretely specified polyethylenes is available with density-modulus combinations which range from HDPE through LLDPE to

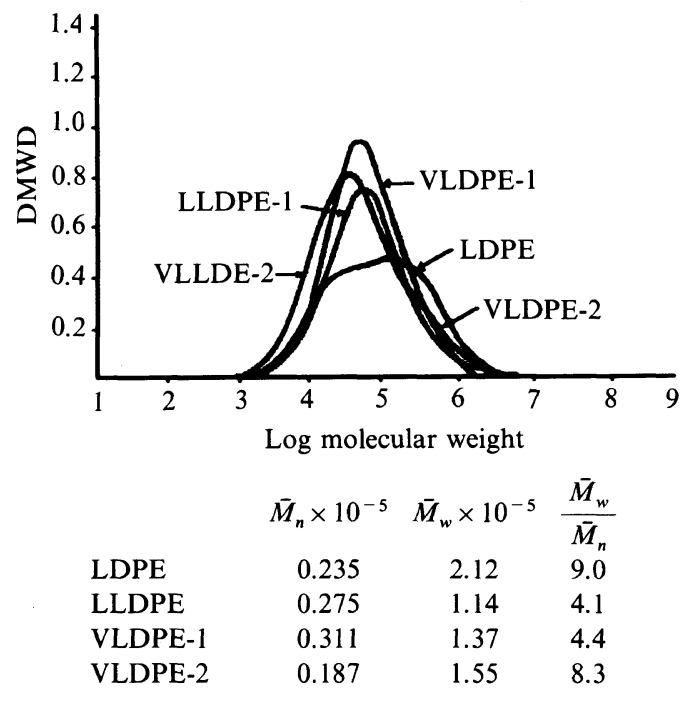

Figure 1. Molecular weight distribution of typical polyethylenes (courtesy of Dr. S. J. Kurtz ${ }^{1}$ ).

VLDPE and ultimately to ethylene-propylene rubbers. These new grades have the potential to displace PVC, polyethylene vinyl acetate and polyurethanes in a variety of applications. No doubt there will continue to be a steady evolution of more advanced products even in this mature market, all of which will stem from research on high mileage heterogeneous catalysts and developments in process technology.

\section{MACROSCOPIC POLYMER STRUCTURES}

In the field of polymer composites and other two-phase systems, control of the macrostructure is more important than control of the 
polymer chain microstructure. In the last decade fibre-reinforced polymer composites have incorporated new matrix materials and new fibres. Thus to epoxy and urethane matrices have been added higher performance poly aryl sulphones and polyether ether ketones (PEEK). Glass fibres are now in competition with higher modulus organic fibres such as KEVLAR and also with carbon fibre. In terms of the components of the composites the next decade may not see much change; more likely the emphasis will fall on new methods of assembly. Surely the major change must come in the management of the fibre orientations or lay-up during the formation of the composite component. Except for simple geometrics such as fibres oriented along the axis of a rod, or complex air frame sections which command a high price, the manufacturing technology to realize the full potential of anisotropic properties in ready-formed components is not available. The prospects for the substitution of metal structural components by reinforced plastics awaits improved fabrication technology and thus will be a spur for industrial research in this field.

In another form of 'composite,' foams, there is prospect of improved control over the macroscopic structure. Polystyrene and polyurethane foamed structures are readily available; the foams are formed either by generation of gas during the polymerization or by phase separation of the polymer from solutions. They can be produced with open or closed cells and at densities 5-10 times lower than the bulk material. Both rigid and flexible structures are well known but the cellular geometry is highly irregular.

However, a novel method for the production of porous materials with more regular cellular structure has been developed using high internal phase emulsions as templates. ${ }^{2}$ The monomer, for example styrene, is incorporated in the continuous phase in an aqueous emulsion stabilized by surfactants, as shown schematically in Figure 2.
POLYHIPE POLYMER

The emulsion route to porous polymers

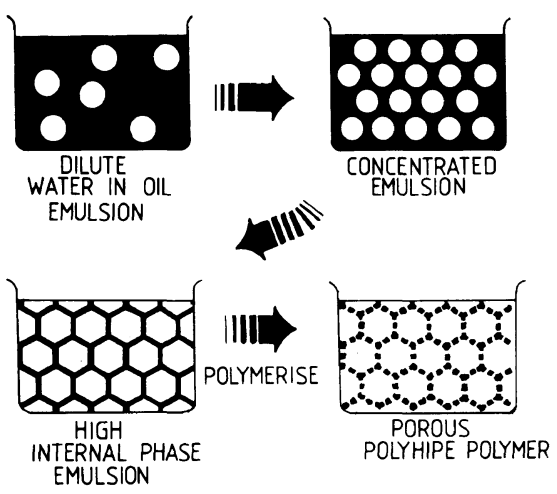

Figure 2. Schematic description of the emulsion route to porous polymers.

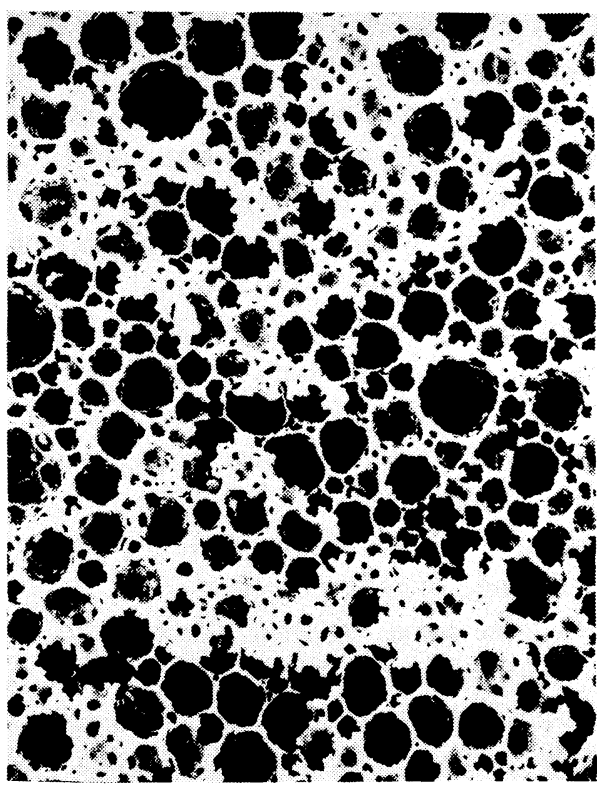

Figure 3. Photograph of polystyrene porous polymer produced via the emulsion route. (Magnification $2 \mathrm{~K}$ )

Conventional emulsion technology allows the droplet size and the range of droplet sizes to be controlled at this stage of the process. Polymerization of the monomer yields a continuous porous polymer structure containing droplets of water. If the cell structure is open the water is easily removed to give a foam of the corresponding structure. Cell sizes down to 1 micron can be generated and a scanning 
Table II. Mechanical properties of some polystyrene polyhipe polymers

\begin{tabular}{|c|c|c|c|}
\hline Density & idage & Modulus & Cell size \\
\hline $\mathrm{g} \mathrm{cm}^{-3}$ & (porosity) & $\mathrm{Nm}^{-2} \times 10^{-7}$ & $\mu \mathrm{m}$ \\
\hline 0.17 & 82 & 2.49 & $20-25$ \\
\hline 0.14 & 86 & 1.83 & $20-25$ \\
\hline 0.10 & 90 & 1.14 & $20-25$ \\
\hline 0.07 & 93 & 0.73 & $20-25$ \\
\hline 0.04 & 97 & 0.37 & $20-25$ \\
\hline
\end{tabular}

electron micrograph of a typical structure is shown in Figure 3. Coherent structures can be formed in emulsion containing as little as $3 \%$ by volume of the continuous phase.

The technique can be used to make porous polymers of controlled structure from any incompatible monomer/solvent or pre-polymer/solvent system. Crystalline, plastic and rubbery materials of high porosity can be generated which have good micro filter properties and good dimensional stability. Hydrophilic polymers which swell in water generate super-absorbent materials. Additionally, block copolymer structures can be generated in which different regions have different characteristics. Table II gives some properties of open-cell porous polystyrene over a range of bulk densities.

It will be apparent from the smooth graduation in properties down to very low densities that good structural control is achieved. Clearly this range of materials opens up the prospect of advanced structures from commodity polymers for application in separation technology to chemical supports and ultra lightweight components.

\section{NATURALLY OCCURRING POLYMERS}

\section{Modified Natural Rubber}

It may be surprising that prospects for advanced polymer research exist in such a mature field. However, work by the Malaysian
Rubber Research Board on the epoxidation of natural rubber in the fresh latex state, shows that there is still scope for simple, controlled chemical modification of natural rubber at source. $^{3}$ The products have the potential to become fairly high tonnage materials with specific attributes.

Careful control of the reaction conditions in rubber latex using $\mathrm{H}_{2} \mathrm{O}_{2}$ /acetic acid gives selective epoxidation of natural rubber with little or no side reaction. Products which incorporate 10,25 , and $50 \mathrm{~mol} \%$ of epoxide have been examined in some detail.

Epoxidized Natural Rubber

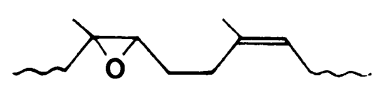

The incorporation of epoxide units in the 10 $\mathrm{mol} \%$ material has been shown by NMR spectroscopy to be random. A survey of properties is given in Table III, where it is shown that properties such as $T_{\mathrm{g}}$, resilience and gas permeability change smoothly with degree of substitution.

The materials all shown strain crystallization and so modulus and tensile strength are not dissimilar to natural rubber. The $50 \mathrm{~mol} \%$ rubber looks particularly interesting having oil resistance similar to nitrile rubber and having gas permeability as low as butyl rubber. Figure 4 shows the potential of the $25 \mathrm{~mol} \%$ material in tyre treads, with epoxy natural rubber compounds being superior in both rolling resistance and wet grip to current materials.

There appears also to be great potential for these epoxy rubbers as metal adhesives. Finally, the simplicity of the process technology offers the prospect of a not-tooexpensive material. Clearly there is scope for other chemical conversions to add value to natural rubber; the need is for precise chemistry of elegant simplicity.

\section{Modified Polysaccharides}

There is a well developed chemical tech- 
Table III. Physical properties for some epoxidated natural rubbers (ENR)

\begin{tabular}{lcccc}
\hline & NR & ENR-10 & ENR-25 & ENR-50 \\
\hline$T_{\mathrm{g}} /{ }^{\circ} \mathrm{C}$ & -75 & -60 & -45 & -28 \\
Black filled vulcanizate & & & & \\
$\quad$ properties & 7.7 & 8.0 & 7.1 & 7.4 \\
$300 \%$ Modulus/MPa & 27.0 & 25.4 & 24.8 & 23.3 \\
Tensile strength/MPa & 78 & 76 & 40 & 25 \\
Resilience $23^{\circ} \mathrm{C} / \%$ & 78 & - & 40 & 1 \\
Oil swelling ASTM No. 3 & 100 & - & 32 & 12 \\
4 days at $23^{\circ} \mathrm{C} / \%$ & & & \\
Relative air permeability & & & & \\
\hline
\end{tabular}

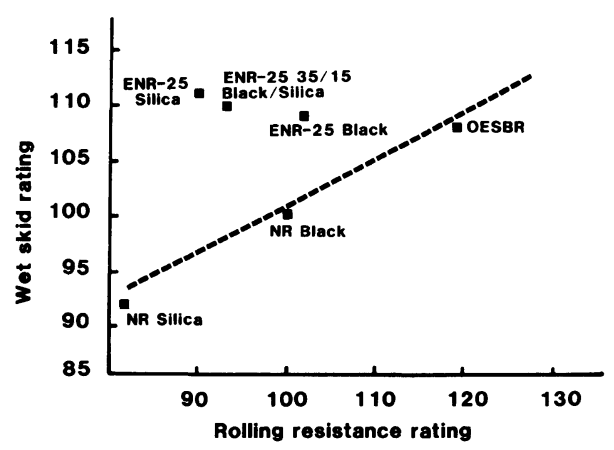

Figure 4. Wet grip rating vs. rolling resistance for steel radial tyres treated with epoxidated natural rubbers (ENR) as indicated (courtesy Dr. I. R. Gelling ${ }^{3}$ ).

nology for the modification of polysaccharides obtained from vegetable matter; for example the solubility and the viscosity of cellulosic materials can be usefully modified by carbo methylation. Biotechnology offers routes to a more specific tailoring of polysaccharide structures to give industrially desirable and reproducible enhancement of properties. ${ }^{4}$ These materials are used as thickeners in aqueous systems and often out-perform synthetic polymers; for example 1-4 linked polysaccharides have $[\eta] / \mathbf{M}$ ratios considerably greater than for synthetic polymers. $\eta_{\mathrm{sp}}$ is high even at low concentrations. The polymannose and cellulose structures typical of this class of natural polymers are shown in Figure 5. These structures are insoluble in water, but solubility is improved when the $-\mathrm{CH}_{2} \mathrm{OH}$ side groups along the D-mannan (M) backbone are sub-
(I)

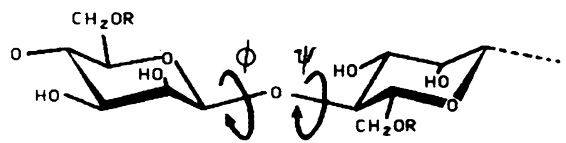

$(R=H$ in some residues and $\alpha D$-galactosyl in others)

(II)

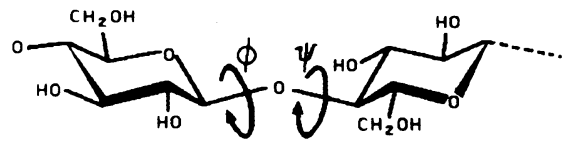

Figure 5. Comparison of the primary structure of guar galactomannan(I) with cellulose(II).

stituted with galactosyl residues $(\mathrm{G})$

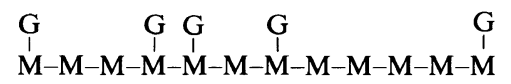

Guar gum is one of the most commonly available galactomannans, but is oversubstituted so far as optimal proportions are concerned. Considerable research effort in industry is now directed towards enzymatic modification of these gums so that galacttse units are selectively removed by geometrically engineered enzymes to give controlled structures. Removal of galactose units reduces the solubility and enhances intra molecular aggregation of the polysaccharides giving rise to higher viscosity per gram of polymer. These developments will open up prospects of materials of reproducible specification in a field previously regarded as unamenable to 'high' polymer technology. 


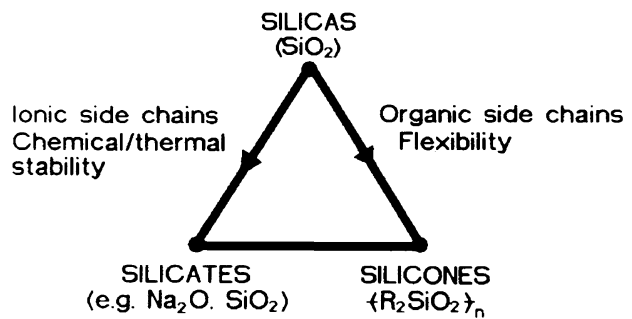

Figure 6. Schematic diagram for various polysiloxanes.

\section{Inorganic/Organic Hybrid Polymers}

Siloxanes apart, the field of inorganic polymers has yielded disappointing returns despite the intense search in the 50's and 60's for inorganic backbones which would confer thermal stability. Hydrolytic instability has usually stood in the way of industrial development. However, many naturally occurring minerals including phosphates and silicates have essentially linear, ladder or sheet-like polymer structures. Some current research is directed towards hybrid structures of inorganic polymers with organic features in the hope of obtaining materials which combine the characteristics of both components, e.g., toughness and flexibility.

Some work has concentrated on phosphate glasses to produce materials of lower melting point and flexibility but water resistance is a major problem. Current efforts concentrate on the silica, silicate, silicone systems (Figure 6).

Acid leaching of silicate minerals, followed by silylation of the silicic acid residues generates organophilic polymers. ${ }^{5}$ Further development of the chemical functionality offers scope for new polysiloxane-silicate hybrids. An alternative strategy incorporating $\mathrm{N}$ and $\mathrm{Al}$ in these essentially $\mathrm{Si}, \mathrm{O}, \mathrm{C}$ structures would build an industrially important bridge to the new ceramic materials which are now being developed actively in Japan, Europe and U.S.A.
Table IV. Approximate selling prices of speciality polymers

\begin{tabular}{lr} 
& $\$ / \mathrm{lb}$ \\
\hline UHMW Polyethylene & 1.0 \\
Polyacetal & 1.5 \\
Polycarbonate & 1.6 \\
Poly(phenylene oxide) & 2.0 \\
Poly(ester carbonate) & 2.5 \\
Poly(ether imide) & 4.3 \\
Poly(ether sulphone) & 4.2 \\
PTFE & 5.0 \\
PDMS & 5.5 \\
Poly(vinylidene fluoride) & 6.0 \\
Poly(amide imide) & 14.0 \\
Poly(ether ether ketone) (aryl) & 30.0 \\
Reference: Polyethylene & $0.3-0.4$ \\
\hline
\end{tabular}

\section{SPECIALITY POLYMERS}

The past decade has been notable for the growth in importance as speciality polymers relative to high-tonnage commodity products. Although speciality polymers account only for about $10 \%$ of the total tonnage, they contributed $25 \%$ of profit in 1984 and it is estimated that current growth in this market is double that of commodity polymers. Table IV gives an idea of the range of commodity polymers available and their relative price.

It is in this area of polymer technology where one expects the brightest prospects for advanced polymer research in industry. There has been already a change in emphasis and as we shall see it may culminate in multidisciplinary polymer research in the broadest context so far envisaged.

\section{Current Position}

By 1970 there were essentially three wellestablished speciality polymers, viz:-

PTFE $\left(\mathrm{CF}_{2} \cdot \mathrm{CF}_{2}\right)_{n}$, chemically inert, solvent resistant, high melting very low coefficient of friction,

Polydimethylsiloxane $\left(-\mathrm{Si}\left(\mathrm{CH}_{3}\right)_{2} \mathrm{O}\right)_{n}$, very low $T_{\mathrm{g}}$, thermally stable high resilience rubbers, 
Polyvinylidenefluoride $\left(\mathrm{CH}_{2} \cdot \mathrm{CF}_{2}\right)_{n}$, large piezoelectric coefficient.

In the 70's a second wave of speciality polymers appeared. They possessed good

The group includes:

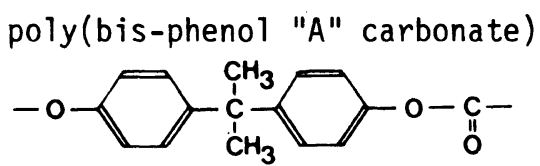

several poly suiphones, e.g.

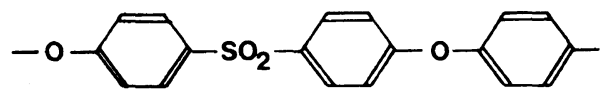

poly(2,6-di-methylphenylene oxide)

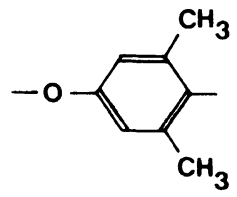

The work on high temperature resistant polymers has concentrated on paraphenylenelinked systems so far as engineering plastics are concerned but the more intractable polyimide and benzimidazole hetero cyclic systems have been used for very high temperature stability.

Perhaps the most important clue to the direction of future research is given by the high modulus fibres of this group of materials. KEVLAR is one of the most highly developed fibres in this class, $(\mathrm{CO}-\mathrm{O}-\mathrm{NH})_{n}$, and the important feature is that it undergoes a degree of self-orientation at the molecular level in solution and in the melt, prior to drawing.

On into the 80 's a considerable family of adhesives based on precise formulations has been added to the list of speciality polymers. Photoresists for the microelectronics industry and a wide range of polymers and speciality fabricated polymer components have been developed for biomedical applications.

There are two clear features of the present industrial position.

(i) in almost every new application con- engineering properties such as toughness, stiffness, high tensile strength and temperature resistance up to $c a .200^{\circ} \mathrm{C}$.

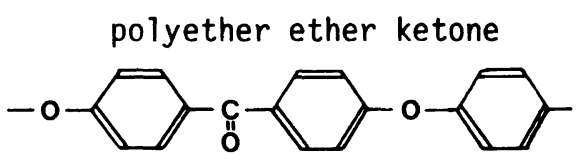

polyether imide

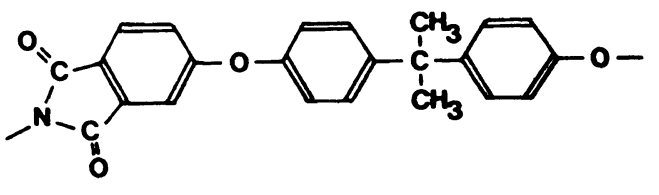

ventional polymer synthetic routes are used, albeit on more complex monomers or mixtures of monomers and,

(ii) the desired properties are usually achieved by adjustment of the chemical structure in essentially homopolymer systems, i.e., by deeigning polymers with specific functionalities.

Most of the random copolymers or blends on the market are designed to give a range of properties intermediate between those of the present homopolymers rather than to meet selected high performance criteria. Block copolymers present a different picture because physical processing can order the two-phase structures and develop unique anisotropic properties over and above the properties conferred by chemical structure.

\section{Future Prospects}

This observation together with the capacity for self orientation shown by poly(parabenzamide) structures provides one pointer to new directions of advanced polymer research in industry. Chemical functionality 
will be allied to physical organization at the molecular level often at the prepolymer stage to provide new materials. Order can be generated through inherent characteristics of molecular structure (e.g., liquid crystal type alignment or phase separation) or by polymerization of monomers at interfaces or in other ordering environments. Optical and electrical properties are now sought after along with thermal and mechanical. For most of these systems the markets are not yet established but the trends are to be seen in university and industrial research laboratories.

\section{(i) Self-Orienting-Polymers}

There are two main routes to self-orientated polymers now being pursued. The first stems from polymerization in the solid (crystalline) state, the aim being to improve the orientation in the monomer crystal on the crystalline polymer generated. Trioxane, for example, was converted to polyformaldehyde in the solid state, unfortunately polymerization actually occurred in small liquid domains and so no significant orientational effects arising from the original crystal structure were retained. However, in the last decade Baughman's group has demonstrated that the orientation of the single crystal of monomer can only be preserved if the subsequent polymer crystal has a density closely similar to that of the monomer crystal. Otherwise the change in volume destroys the molecular selectivity of steric control. In addition there are various aspects of the symmetry of the monomer crystal which must be preserved or changed only according to certain selection rules in the resulting polymer crystal.

The second route, exemplified by Kevlar, is to take advantage of the capacity for selforientation in the melt or in solution which arises from the existence of rigid segments capable of intermolecular and intra molecular association, usually manifest in the formation of thermotropic liquid crystal structures. A great deal of work is going on, principally with paraphenylene linked mesogenic units inserted in the main chain of the polymer structure, ${ }^{6}$ or as pendant side-groups. Orientational effects have been clearly demonstrated in melts and rubbers and in partially crystalline materials of both types. The degree of orientation and the propensity to liquid crystal formation is greatly enhanced in both types of polymer by the use of flexible spacer groups (e.g., n-decamethylene) to allow the mesogenic group sufficient molecular mobility to move into alignment with neighbouring mesogenic groups. Nematic, or smectic liquid crystal phases are frequently encountered in these materials and in polymers (e.g., polyester with triad aromatic ester groups) with mesogenic units in the main chain, cholesteric mesophases are reported. In the polyesters, phase transition from crystalline to cholesteric and thence to the isotropic phase are fully reversible.

These materials provide families of polymers with new optical or electronic properties and could create new markets. But equally the self-orientation could be exploited in new fibre forming materials or as a route to selfreinforcing polymers which might herald a new class of thermoplastic composites.

\section{(ii) Thin Films}

These materials provide families of polymers wiih new optical or electronic proper(Langmuir-Blodgett) films of organic materials for applications in optios or microelectronics is a flourishing area of research sometimes called 'molecular electronics.' Monomolecular films can be formed directly on the surface of the liquid in the trough and a dipping technique enables multi-layer films to be built upon the surface of templates such as microscope slides. An obvious development is the use of organic molecules which contain polymerizable groups to form such films. Subsequent polymerization, for example of $-\mathrm{C} \equiv \mathrm{C}-$ by UV irradiation, can provide coherent polymer films of specific molecular thickness ${ }^{7}$ as shown schematically in Figure 7.

There is great promise for both optically and electronically active polymer films by this 


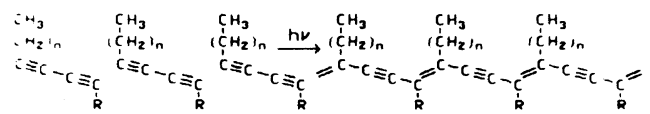

IN MONOLAYERS :

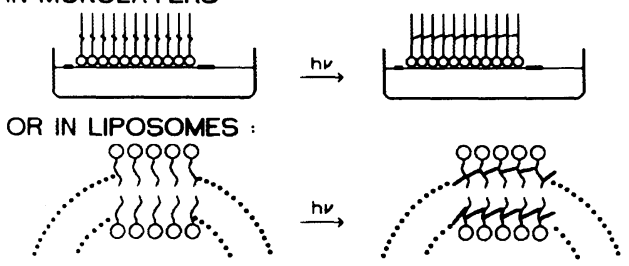

Figure 7. Polymerization of diacetylene lipid (according to Gros et $a l^{7}$ ).

route and in addition it offers the prospect of the controlled synthesis of membranes at a molecular level. There is currently considerable interest in the synthesis of functional vesicle membranes by this route where by ultra sonication of the trough liquid with its layers of monomer spread on the surface produces vesicles which micro encapsulate the liquid. Again UV initiation of the polymerization of $-\mathrm{C} \equiv \mathrm{C}-$ triple bonds produces the vesicle membrane.

(iii) Conducting Polymers

The search for conducting polymers began

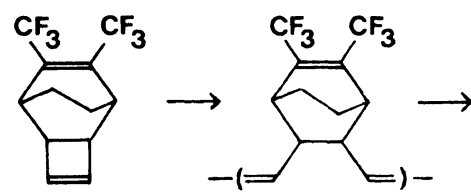

Thin, coherent films of polyactylenes are produced by this chemical route in contrast with the disordered fibrillar mats produced by direct polymerization.

Conductivities approaching those of metallic good conductors are only achieved however by doping highly conjugated polymer structures with electron donor or acceptor compounds. Polyacetylene and its derivatives, polypyrroles and polyphenylenes are amongst polymers which have been transformed into semi-conductors and metallic conductors ${ }^{9}$ by the addition of, for example, $\mathrm{I}_{2} \mathrm{SbF}_{5}$ or $\mathrm{AsF}_{5}$ (cf. Table V).
Table V. Conductivities $\left(\mathrm{s} \mathrm{cm}^{-1}\right)$ for Polymers and Copolymers doped with $\mathrm{AsF}_{5}$

\begin{tabular}{ccrccc}
\hline $\mathrm{A}$ & $\mathrm{B}$ & $(\mathrm{A})$ & $(\mathrm{B})$ & $(\mathrm{AB})$ & $\left(\mathrm{AB}_{2}\right)$ \\
\hline$-\mathrm{HC}=\mathrm{CH}-$ & $-\mathrm{C}_{6} \mathrm{H}_{4}-$ & 1200 & 500 & 3 & - \\
$-\mathrm{C}_{6} \mathrm{H}_{6}-$ & $-\mathrm{C}_{6} \mathrm{H}_{4} \mathrm{~S}-$ & 500 & 1 & 0.3 & 0.02 \\
$-\mathrm{C}_{6} \mathrm{H}_{4} \mathrm{~S}-$ & $-\mathrm{C}_{6} \mathrm{H}_{4} \mathrm{O}-$ & 1 & $10^{-3}$ & $10^{-4}$ & $10^{-6}$ \\
\hline
\end{tabular}

in the early 60's and abortive attempts were made to find configurated systems which would exhibit super conductivity at temperatures above $4 \mathrm{~K}$. More realistic targets are now set and the main effort is to synthesize polymer systems with conductivities approaching those of metals.

Elagant organic synthetic methods are being used to develop polyacetylenes as intrinsic polymer conductors by virtue of their conjugated backbones. ${ }^{8}$ The inherent reactivity of the monomers has lead to a two-stage synthesis for pure polymers. A precursor polymer is formed which is soluble and can be purified. In a final thermal stage the precursor undergoes a retro-Diels Alder reaction to yield the confugated system, as shown below:

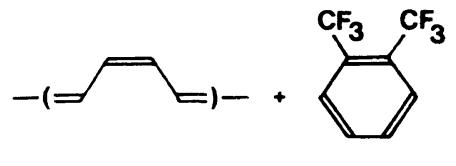

One of the most exciting developments has been the incorporation of low percentages of these charge-transfer polymer conductors in polymer matrices. ${ }^{10}$ Controlled crystallization and solidification of the melt allows the organic conductor to form as a dendritic structure in the rigid polymer matrix.

The market for these prototype materials is expanding steadily. Applications have already been found for organic conductors in photo conductor or pyroelectric devices where high conductivity was not essential and some metal-polymer composites have been used. The real breadth of possible application how- 
ever is still to be explored, as, indeed, is the range of new conducting systems.

\section{ADVANCED POLYMER RESEARCH IN INDUSTRY}

In this review I have tried to emphasize that there is still scope for advanced polymer research to support even the most mature of the high tonnage industrial polymers. Furthermore, there is scope too for adding value to some of the naturally occurring polymers at source, either by simple chemistry or by enzymic modification to yield better defined products.

Of course, the scope in the field of speciality polymers has never been more exciting. For the chemist, exotic monomers may yield polymer properties which can bear the cost of expensive starting materials. So the vista constrained to relatively simple feedstocks in the petrochemical years is suddenly opened up again. There are few signs however of new polymerization reactions. All the materials in this paper rely on conventional polymerization chemistry.

There is however a major issue to be faced when one considers the new materials which rely on pre orientation or self orientation of the monomer system or the properties of thin films or special features of electrical conductivity. Here the materials are being developed in close collaboration with physicists who are designing advanced devices. The polymers become an integral part of the device and perhaps it will be necessary to form them in situ to obtain optimum properties. This could be true equally in some medical or biological applications. Thus multidisciplinary research teams of broader cross sections of skills than have been used hereto can be envisaged. What will the relative contributions from the polymer industry and say the electronics industry be? Certainly polymer science is well used to providing a service as an enabling technology. There will be a need for the industries to interface effectively - maybe by joint ventures. But ultimately this will be resolved perhaps by the polymer industry adopting a strategy which optimises the return on the added value, without becoming directly involved.

\section{REFERENCES}

1. S. J. Kurtz, Plast. Rubber Int., 11, 34 (1986).

2. D. Barby and Z. Haq, Eur. Patent Application 60138 (1982).

3. I. R. Gelling, Rubber Chem. Technol., 58, 86 (1985).

4. B. V. McClearly, I. C. M. Dea, J. Windust, and D. Cooke, Carbohydr. Polym., 4, 253 (1984).

5. See for example, K. Kuroda and C. Kato, Polymer, 19, 1300 (1978).

6. Ho-Jin Park, Jung-Il Jin, and R. W. Lenz, Polymer, 26, 1301 (1985).

7. L. Gros, H. Ringsdorf, and Schapp, Angew. Chem., Int. Ed. Engl., 20, 305 (1981).

8. J. H. Edwards and W. J. Feast, Polymer, 21, 595 (1980).

9. R. H. Baughman, J. L. Bredas, R. R. Chance, R. L. Elsenbaumer, and L. W. Shacklette, Chem. Rev., 82, 209 (1982).

10. M. Kryszewski, J. K. Jeszka, J. Ulanski, and A. Traez, Pure Appl. Chem., 56, 355 (1984). 